\title{
28 Research Square \\ Development and Verification of the Amino Metabolism Related and Immune-associated Prognosis Signature in Gliomas
}

\author{
Yang Xu \\ Renmin Hospital of Wuhan University: Wuhan University Renmin Hospital \\ Liguo Ye \\ Renmin Hospital of Wuhan University: Wuhan University Renmin Hospital \\ Rongxin Geng \\ Renmin Hospital of Wuhan University: Wuhan University Renmin Hospital \\ Ping $\mathrm{Hu}$ \\ Renmin Hospital of Wuhan University: Wuhan University Renmin Hospital \\ Qian Sun \\ Renmin Hospital of Wuhan University: Wuhan University Renmin Hospital \\ Shiao Tong \\ Renmin Hospital of Wuhan University: Wuhan University Renmin Hospital \\ Fanen Yuan \\ Renmin Hospital of Wuhan University: Wuhan University Renmin Hospital \\ Qianxue Chen ( $\square$ chenqx666@whu.edu.cn ) \\ Wuhan University Renmin Hospital https://orcid.org/0000-0002-9413-1030
}

\section{Primary research}

Keywords: gliomas, gene signature, amino acid metabolism, prognosis, microenvironment, immune

Posted Date: August 3rd, 2021

DOI: https://doi.org/10.21203/rs.3.rs-702786/v1

License: (1) (1) This work is licensed under a Creative Commons Attribution 4.0 International License. Read Full License 


\section{Abstract}

Background: Aberrant reprogramming of metabolism has been considered as a hallmark in various malignant tumors. The metabolic changes of amino acid not only have dramatic effects in cancer cells but also influence their immune-microenvironment in gliomas. However, the features of the amino acid metabolism-related and immune-associated gene set haven't been systematically described.

Methods: The expression level of mRNA was obtained from TCGA database and CGGA database, which was used as training set and validation set respectively. Different bioinformatic and statistical methods were combined to construct a robust amino metabolism related and immune-associated risk signature for distinguishing prognosis and clinic-pathological features. Constructing the nomogram enhanced risk stratification and quantify risk assessment on the basis of our gene model. Besides, the biological mechanism related to the risk score was investigated by gene set enrichment analysis. Hub genes of risk signature were identified by PPI network.

Results: The amino acid metabolism-related and immune-associated gene signature recognized high-risk patients defined as an independent risk factor for overall survival. The nomogram exhibited high accuracy of predicting OS rate for glioma patients. Furthermore, high-risk score hinted immunosuppressive microenvironment and lower sensitivity of ICB therapy and also identified PSMC5 and PSMD3 as novel biomarkers in glioma.

Conclusion: A novel amino acid metabolism-related and immune-associated risk signature for predicting prognosis in glioma has been constructed and identified two potential novel biomarkers.

\section{Introduction}

Metabolic reprogramming is critical for maintaining the survival of cancer cells and defines as a hallmark of cancer, which might be the consequence of oncogenic mutations [1]. Amino acid metabolism also emerges as an important role in the metabolic reprogramming of cancer cells, because its function of redox balance, energy regulation, biosynthesis support and so on [2]. Amino acids and its derivatives can not only regulate cancer cells but also modulate the surround microenvironment, which enhances the malignancy and immunosuppression of tumor [3]. For instance, arginine-derivations could change chromatin structure to regulate gene expression, which promotes the proliferation of cancer cells [4]. Kynurenine, which is the catabolic products of tryptophan, induces the invasion of cancer cells and immunosuppression of tumor microenvironment [5] by binding to transcription factor aryl hydrocarbon receptor (AHR) [6]. Moreover, the activation of AHR hampers the performance of dendritic cells and T cells, which play a role in anti-tumor [7]. Metabolism of amino acid is virous in tumors and plays a significant role not only in the biological process of tumor cells but also in the tumor microenvironment, particularly the modulation of immune. All these indicates a better understanding of the metabolism of amino acids will offer potentially effective targets for cancer therapy [8].

In our study, we focus on glioma which is a group of highly heterogeneous neurocutaneous tumors, accounting for about $26 \%$ of all intracranial tumors, and is the most deadly primary malignant type of brain tumor in adult [9]. Although combined therapy has been developed including precise surgical resection, adjuvant radiotherapy and temozolomide chemotherapy, the overall survival still remains poor and hasn't been significantly improved, furthermore, long-term survival is rare [10]. Although immunotherapy has made break through progress in the treatment of a variety of solid tumors, the specific effect of immunotherapy in glioma is still not clear [11]. It has been found that the expression level of immunosuppressive factors such as PD-L1 and IDO/TDO were dramatically elevated in gliomas. As is well-known, PD-L1 could limit function of effect T cells and the metabolite mediated by IDO/TDO promote the development of immunosuppressive microenvironment $[12,13]$. In addition, the upregulation of Treg cells could exhausting cytotoxic $T$ cells to reduce the damage of tumor cells and enhanced the immunosuppressive effects in glioma microenvironment[14]. However, how amino acid metabolism influence prognosis and immune process in glioma progression need further systematic research.

In our study, the amino acid metabolism-related and immune-associated risk signature was defined as an independent risk factor for the prognosis of glioma patients. The decision tree strongly verified the risk-dependent subgroups, and the nomogram showed extremely high accuracy. In addition, high-risk score hinted immunosuppressive microenvironment and lower sensitivity of ICB therapy and PSMC5 and PSMD3 were identified as novel biomarkers in glioma. In summary, we demonstrated a novel amino acid metabolism-related and immuneassociated risk signature for predicting prognosis for patients with glioma and identified two potential novel biomarkers.

\section{Materials And Methods}

The workflow of our analysis is shown in Figure 1 and specific details are explained in the following sub-sections.

\section{Data preparation and collection}


The expression of mRNA and clinical information of patients were collected from 698 patients in the cancer genome atlas database, consistently, 413 samples were collected from 693 samples in Chinese Glioma Genome Atlas part B as a validation set. Moreover, the mRNA data of normal brain tissue was collected from Genotype Tissue Expression project (GTEx).

\section{Obtaintion of amino metabolism related genes}

Amino acid metabolism-related gene sets (REACTOME_METABOLISM_OF_AMINO_ACIDS_AND_DERIVATIVES) were obtained from the Molecular Signatures Database v5.1 (MSigDB) (http://www.broad.mit.edu/gsea/msigdb/).

\section{Determination of the Immune Status Through single sample gene set enrichment analysis}

Using single sample gene sets enrichment analysis (SSGSEA) algorithm based on the transcriptome profiling data and corresponding immunity-related gene sets retrieved from MSigDB $[15,16]$ and using ESTIMATE algorithm analyzed the estimation of Stromal and Immune cells in Tumor tissues [17], which has been developed to measure stromal level (stromal score), cyto-infiltration degree (immune score) and tumor purity.

\section{Construction of amino metabolism related and Immune-Associated signatures}

The R package 'WGCNA' was used to construct a scale-free co-expression network to verify a gene module which is mostly related to amino metabolism and Immune in glioma. To explore the most robust genes, LASSO regression model was performed[18]. Furtherly, the risk scores were calculated by multiplying gene expression by the regression coefficient acquired upon Lasso regression. Based on the median risk score, all cases were divided into high- or low-risk group.

\section{Prognostic value and TICs Profile of risk model}

The prognostic significance of the risk signature was evaluated by Kaplan-Meier survival curves. And independent prognostic factors including the risk score in glioma were investigated by univariate and multivariate Cox regression analyses. Subsequently, investigate the specificity and sensitivity of risk score in the prediction of 5-year overall survival (OS) by analyzing the receiver operating characteristic (ROC) curve [19]. Next, a nomogram according related prognostic factors was constructed to quantitatively predict the 1 -, 2 - and 3 -year survival rate in glioma patients. The abundance profile of TICs was estimating by CIBERSORT [20]. computational method in low and high-risk group to explore the correlation of the TICs in different groups

\section{Gene Set Enrichment Analysis}

In Molecular Signatures Database, Hallmark and C7 gene sets were downloaded, which was used as the target gene sets to investigate the gene sets associated with risk score in whole transcriptome of all glioma samples in TCGA performed by the software GSEA-3.0. (NOM $p<$ 0.05 and FDR $q<0.05$ were considered significant).

\section{Functional Annotation for Genes of Interest and Construction of PPI}

To explore Gene Ontology (GO) of selected genes, R package cluster Profiler package was used to explore the functions among genes of interest, with a cut-off criterion of adjusted $p<0.05$. GO annotation that contains the three sub-ontologies- biological process (BP), cellular component (CC), and molecular function (MF)-can identify the biological properties of genes and gene sets for all organisms [21]. Online tool STRING (Search Tool for the Retrieval of Interacting Genes) was used to predict protein-protein interactions (PPI) and construct a PPI network of selected genes. Using the STRING database, genes with a score 0.4 were chosen to build a network model visualized by Cytoscape (v3.7.2) [22] . In a co-expression network, Maximal Clique Centrality (MCC) algorithm was reported to be the most effective method of finding hub nodes [19]. The MCC of each node was calculated by CytoHubba, a plugin in Cytoscape [23]. In this study, the genes with the top 10 MCC values were considered as hub genes.

\section{Verification of the Expression Patterns and the Prognostic Values of Hub Genes}


To explore the potential reliability of the hub genes, the expression level of each hub gene between cancer and normal tissue was plotted as a box plot graph. On the basis of TCGA database, Kaplan-Meier univariate survival analysis was performed by using the survival package in $\mathrm{R}$ software to explore the relationship between overall survival and disease-free survival with hub genes in patients. In the study, all the patients selected for survival analysis should be with complete clinical information, consequently, on the basis of median expression value of hub genes, these samples divided into two subgroups. The survival-related hub genes with log-rank $p<0.05$ were regarded as statistically significant.

\section{Human tissue samples}

The normal brain tissues were collected from the patients who suffered from serious brain injury. The glioma samples were obtained from the Department of Neurosurgery, Renmin Hospital of Wuhan University, Wuhan, China. The clinical glioma specimens were examined and diagnosed by pathologists at Renmin Hospital of Wuhan University. This study was approved by the Institutional Ethics Committee of the Faculty of Medicine at Renmin Hospital of Wuhan University (approval number: 2012LKSZ (010) H). Informed consent was obtained from all patients whose tissues were used.

\section{RNA extraction and quantitative real-time PCR}

Total RNA of glioma tissues was extracted using by Trizol reagent (G3013-100ML, Servicebio, Wuhan, China), and cDNA was synthesized by SweScript RT I First Strand cDNA Synthesis Kit (G3330-50; Servicebio, Wuhan, China). Quantitative real-time PCR (qPCR) for PSMC3 and PSMD5 mRNA levels were performed using SYBR qPCR SuperMix (E096-01B, novoprotein, China) according to the manufacturer's instructions and performed in Bio-Rad CFX Manager 2.1 real-time PCR Systems (Bio-Rad, Hercules, CA, USA).

GAPDH was set as internal controls and relative Ct method was used to analyze the data. The sequence of primers were listed in table S3.

\section{Results}

\section{Construction of Weighted Gene Co-expression Modules}

First, combining the mRNA data in TCGA-glioma and GTEx database, 10, 550 differentially expressed genes between glioma and normal brain tissue were detected. Among these genes, 260 amino acid metabolism related genes were ensured (Figure S1A). KEGG and GO analysis confirmed that these nods were mainly related to the biological function and pathway of amino acid metabolism, PPI network showed a strong co-expressed correlation among the genes (Fig. S1B-D). Then, samples in the training set were hierarchically clustered in immunityhigh (Immunity-H) or immunity-low (Immunity-L) group by ssGSEA (Figure S2A, B). Boxplot of immune cells' fraction in glioma tissues were significantly different among immunity-H and immunity-L groups (Figure S2C). Consistently, stromal scores, immune scores and ESTIMATE scores of glioma samples in the Immunity-H group remarkably increased compared with those in the Immunity-L group (Figure S2D-F), meanwhile, the tumour purity in Immunity-H was significantly lower than in Immunity-L group (Figure S2G). Besides, patients in Immunity-H group had a significantly poorer prognosis than other groups (Figure $\mathrm{S} 2 \mathrm{H}$ ).

To find the correlation between amino acid metabolism-related genes and immune infiltration in TCGA-glioma, the gene co-expression networks were constructed from the TCGA glioma datasets with the WGCNA package. 2 modules in the TCGA-glioma were recognized and assigned different colors with each module (Fig. 2A). Then, we created a heatmap of module-immune relationships to evaluate the association between each module and different immune score (high and low). The results of the module-immune relationships showed that the grey module had the highest association with immune-high group (pink module: $r=0.27, p<0.001$ ) in TCGA-glioma (Fig. 2B). The module membership and gene significance were highly correlated in grey module (Fig. 2C).

\section{Identification of a 12-gene risk signature associated with amino acid metabolism and immune in glioma}

To identify the amino acid metabolism-related and immuno-associated risk signature, the univariate Cox regression analysis was used to select 30 genes in the training set, which were related with the prognosis of patients (Fig. 3A). Thereafter, the most relevant biomarkers for prognosis were identified through the LASSO Cox regression model and overfitting was counteracted by ten-fold cross-validation. As a result, the group of 12 genes (PSMC5, GLUD1, DHTKD1, OGDH, PSMF1, PSMD3, PSMB8, PSMB9, PSMD5, PSMD12, PSMC1, PSMD6) were extracted according to LASSO coefficients (Fig. 3B, C). The median of risk score was defined as the cutoff value to divide training set into two 
subgroups including high-risk and low-risk group, and the significant difference was found in both molecular and clinical characteristics between these subgroups (Fig. 3D).

At the same time, there were significant differences according to the risk signature values of age-stratified and WHO grade-stratified clinical samples in both TCGA and CGGA cohorts (Fig. 4A, B, E, F). The molecular pathological diagnosis of glioma has been put forward in clinical practice, IDH wild type and 1p19q non-codeletion gliomas were all the poor prognostic factors and had an inadequate response to traditional radiotherapy or chemotherapy of glioma patients [24]. Such being the case, the distribution of the 12-gene signature was explored based on IDH status-stratified clinical samples were (Fig. 4C, G) and 1p/19q codeletion status (Fig. 4D, H). Overall, these results indicated that the risk score base on the gene signature was significantly associated with clinical features.

\section{Development of the Risk Score Signature and Assessment of the Predicting Capacity}

Based on groups of high-risk and low-risk score, Kaplan-Meier analysis was performed and showed that patients with high-risk score had dramatically reduced overall survival comparing with patients with low-risk score in both TCGA and CGGA dataset (Fig. 5A, B). Besides, as far as 1-year, 3-year and 5-year overall survival, the values of area under the curve (AUC) of ROC curve for TCGA glioma cohort were 0.875, 0.933 and 0.854. Consistently, with regard to 1-year, 3-year and 5-year overall survival, the values of area under the curve (AUC) for CGGA cohort were $0.641,0.678$ and 0.687 , respectively (Fig. 5C, D). Furtherly, the plots were listed to show the distributions of gene expression, risk score and survival status basing on the amino acid metabolism related- and immune-related signature in TCGA and CGGA (Fig. 5E, F). To furtherly explore the significance of our model in evaluating prognosis independently, we performed univariate analysis as well as multivariate analysis, which showed that the value of the risk score might defined as an independent factor to evaluate the prognosis of glioma patients in both TCGA and CGGA (Table 1).

Table 1

Univariate and Multivariate Cox Regression Analyses of Clinicopathologic Characteristics Associated with Overall Survival in TCGA dataset and CGGA dataset

\begin{tabular}{|c|c|c|c|c|c|c|c|c|c|c|c|c|}
\hline \multirow[b]{3}{*}{ Variables } & \multicolumn{6}{|c|}{ TCGA Dataset } & \multicolumn{6}{|c|}{ CGGA Dataset } \\
\hline & \multicolumn{3}{|c|}{ Univariate analysis } & \multicolumn{3}{|c|}{ Multivariate analysis } & \multicolumn{3}{|c|}{ Univariate analysis } & \multicolumn{3}{|c|}{ Multivariate analysis } \\
\hline & $\mathrm{HR}$ & $95 \% \mathrm{Cl}$ & $\begin{array}{l}\mathrm{P} \text { - } \\
\text { value }\end{array}$ & $\mathrm{HR}$ & $95 \% \mathrm{Cl}$ & $\begin{array}{l}P \text { - } \\
\text { value }\end{array}$ & $\mathrm{HR}$ & $95 \% \mathrm{Cl}$ & $\begin{array}{l}P \text { - } \\
\text { value }\end{array}$ & $\mathrm{HR}$ & $95 \% \mathrm{Cl}$ & $\begin{array}{l}\mathrm{P} \text { - } \\
\text { value }\end{array}$ \\
\hline Grade & 4.987 & $\begin{array}{l}3.873- \\
6.421\end{array}$ & $\begin{array}{l}< \\
0.001\end{array}$ & 2.059 & $\begin{array}{l}1.530- \\
2.772\end{array}$ & $\begin{array}{l}< \\
0.001\end{array}$ & 2.635 & $\begin{array}{l}2.353- \\
2.952\end{array}$ & $\begin{array}{l}< \\
0.001\end{array}$ & 2.079 & $\begin{array}{l}1.820- \\
2.374\end{array}$ & $\begin{array}{l}< \\
0.001\end{array}$ \\
\hline Gender & 1.011 & $\begin{array}{l}0.747- \\
1.368\end{array}$ & 0.944 & 0.974 & $\begin{array}{l}0.710- \\
1.336\end{array}$ & 0.870 & 1.022 & $\begin{array}{l}0.870- \\
1.202\end{array}$ & 0.787 & 1.020 & $\begin{array}{l}0.866- \\
1.200\end{array}$ & 0.815 \\
\hline Age & 4.863 & $\begin{array}{l}3.391- \\
6.975\end{array}$ & $\begin{array}{l}< \\
0.001\end{array}$ & 2.296 & $\begin{array}{l}1.465- \\
3.596\end{array}$ & $\begin{array}{l}<.001 \\
0.01\end{array}$ & 1.922 & $\begin{array}{l}1.638- \\
2.256\end{array}$ & $\begin{array}{l}<.001 \\
0.00\end{array}$ & 1.227 & $\begin{array}{l}1.033- \\
1.457\end{array}$ & 0.020 \\
\hline $\begin{array}{l}\text { IDH } \\
\text { mutation } \\
\text { status }\end{array}$ & 0.090 & $\begin{array}{l}0.063- \\
0.129\end{array}$ & $<.001$ & 0.553 & $\begin{array}{l}0.306- \\
1.001\end{array}$ & $\begin{array}{l}<.051 \\
0.05\end{array}$ & 0.367 & $\begin{array}{l}0.315- \\
0.428\end{array}$ & < 0.001 & 0.657 & $\begin{array}{l}0.558- \\
0.773\end{array}$ & $\begin{array}{l}< \\
0.001\end{array}$ \\
\hline $\begin{array}{l}1 p 19 q \\
\text { codeletion } \\
\text { status }\end{array}$ & 0.217 & $\begin{array}{l}0.128- \\
0.370\end{array}$ & $<.001$ & 0.543 & $\begin{array}{l}0.287- \\
1.025\end{array}$ & 0.060 & 0.527 & $\begin{array}{l}0.447- \\
0.621\end{array}$ & $\begin{array}{l}< \\
0.001\end{array}$ & 0.781 & $\begin{array}{l}0.661- \\
0.923\end{array}$ & 0.004 \\
\hline $\begin{array}{l}\text { Risk } \\
\text { Score }\end{array}$ & 9.425 & $\begin{array}{l}6.355- \\
13.978\end{array}$ & $\begin{array}{l}< \\
0.001\end{array}$ & 2.672 & $\begin{array}{l}1.510- \\
4.729\end{array}$ & $\begin{array}{l}< \\
0.001\end{array}$ & 2.448 & $\begin{array}{l}2.076- \\
2.887\end{array}$ & $\begin{array}{l}< \\
0.001\end{array}$ & 1.364 & $\begin{array}{l}1.131- \\
1.646\end{array}$ & 0.001 \\
\hline
\end{tabular}

\section{Combination of the risk signature and clinicopathological features improves risk stratification and survival prediction}

To better enhance risk stratification of prognosis, we constructed a decision tree through patients with different grade of glioma from TCGA. As a result, the difference of overall survival was obviously observed in subgroups with different risk score (Fig. 6A). Developing individualized treatment for individual glioma patients is necessary, consistently, accessing the potential risk and prognosis for individual 
glioma patients is also important. Consequently, we built a nomogram with risk score as well as clinic-pathological features including IDH mutation and 1p19q (Fig. 6D). Besides, the calibration analysis was performed to elevate the accuracy of our nomogram. And the results showed the prediction line of the nomogram was extremely close to the ideal performance (45-degree dotted line) (Fig. 6B, C).

\section{The Differences in Immunocyte Infiltration Degree and enrichment plots of immune related gene sets from gene set enrichment analysis between High- and Low-Risk TCGA Cohorts}

Next, to explore whether our risk score partly accessed the immune status of tumor microenvironment, the relationship of amino acid metabolism and immune related gene signature with the immunocyte infiltration degree was explored in gliomas. Interestingly, our results indicated that $\mathrm{M} 2$ (Cor $=0.31 ; \mathrm{p}=8.8 \mathrm{e}-6)$ and Tregs (Cor $=0.169 ; \mathrm{p}=0.0093)$ were obviously positive related to risk score $($ Fig. $7 \mathrm{~A}, \mathrm{~B})$. Furtherly, NK cells (Cor $=-0.39 ; p=1.9 e-08)$ and CD4 + T cells (Cor $=-0.24 ; p=0.00058)($ Fig. $7 C, D)$ showed negative correlation with the risk score.

Immunotherapy is increasingly becoming an important part of tumor therapy and can significantly improve the prognosis of cancer patients in a variety of solid tumors [25]. Hence, we detected the expression of immune checkpoint in subgroups with high- or low-risk score. According to our gene model, the expression level of PD- L1, PD-1 and CTLA-4 was lower in the glioma patients with high-risk score (P< 0.05$)$ (Fig. 7E, F). This result showed that high risk score group may be less sensitivity to immunotherapy. Furtherly, glioma with high-risk score was obviously enriched in downregulation of the effect immunity pathway, for instance, negative regulation of T cell migration and tumor necrosis factor function (Fig. 7G, H), which indicates an immunosuppressive microenvironment.

\section{Identification of Hub Genes from risk signature as biomarkers in glioma}

The PPI network among the overlapped genes was established by using the STRING database and performed GO and KEGG (Figure S3). MCC algorithm of CytoHubba plugin was used to select hub genes of PPI network and the hub genes were listed in TableS1. Basing on the MCC scores, we selected the top ten highest-scored genes from hub genes, including ODC1, OAZ2, PSMD2, PSMD12, PSMC1, PSMC5, PSMD3, PSME3, PSMD10, PSMD5. The expression levels of these genes were verified according to TCGA database. Kaplan-Meier plotter and the expression level of the top ten genes were performed as shown in Figure S4 and FigureS5. Then, we performed multivariate Cox analysis to evaluate the prognostic value of these genes in gliomas (Fig. 8A and TableS2). In addition, we performed RT PCR in our own clinical samples which includes 6 normal brain tissues, 24 WHO Grade II and 55 GBM samples. Consistently, we found that the expression level of PSMD3 was positive with the grade of glioma, inversely, the expression level of PSMC5 was negative with the grade of glioma (Fig. 8B, D). Moreover, the results of Kaplan-Meier analysis indicated that PSMD3 was significantly associated with worse overall survival of the glioma patients ( $\mathrm{P}$ $<0.05$ ) (Fig. 8C), conversely, PSMC5 was significantly associated with better overall survival of the glioma patients (P<0.05) (Fig. 8E). All the results confirmed that PSMD3 and PSMC5 can be identified as potential biomarkers in glioma patients.

\section{Discussion}

The reprogramming of amino acid metabolism in gliomas has been reported to contribute to the malignancy biological process of glioma including, proliferation, migration and so on. And a previous study has constructed an amino acid-related risk signature for gliomas, which could predict the survival and clinical features of patients [26]. However, more and more studies revealed that amino acid metabolism not only caused by oncogenes alterations but also changed the surrounding tumor microenvironment [27].

In our study, we focused on the amino acid metabolism and immune status which explored by sSGSEA and ESTIMATE, and confirmed differential expression genes in gliomas. Then WGCNA was performed to identify amino acid metabolism and immuno-related gene modules based on the data from TCGA, and amino acid metabolism and immune signature was constructed by

LASSO Cox regression model. Subsequently, the prognostic value of the gene signature was validated in CGGA cohorts. Our risk score system had the ability to distinguish high-risk patients, indicating it can act as a confidential risk factor in complex subgroup of patients. In addition, a decision tree has been constructed to enhance risk stratification on the basis of WHO grade and risk score, which showed that risk score could act as a major determinant. Moreover, the generation of the nomogram was used to quantify risk assessment and survival probability, which could show higher accuracy and discrimination in survival prediction compared with the traditional characteristics.

Furthermore, our risk score could also provide valuable information of glioma microenvironment. In high-risk score subgroup, the infiltration of immunosuppressive cells like Tregs is higher than low-risk score subgroup. Conversely, effect immune-cells are decreased in high-risk score 
subgroup. Consistently, recent study has shown that amino acid metabolism can regulate immune-cells in cancer $[3,28,29]$. Our gene model might provide a clue of how microenvironment was influenced by amino acid metabolism. In addition, immune checkpoint therapy has shown great potential of treatment in diverse solid tumors[30]. However, the therapeutic efficacy has not lived up to expectations in gliomas and the specific mechanisms for the problem still need more research. Different genomic subtypes or molecular profiles are the main challenges in the response to PD-1/PD-L1 checkpoint blockades [31]. In addition, the amino acid derivatives could promote the immunosuppressive microenvironment and even effect the expression of immune checkpoint in glioma [32, 33]. Interestingly, the expression of PD-L1 and CTLA-4 in the high-risk group were significantly lower than the low-risk group in our study. These results indicate better efficacy and greater sensitivity of immune checkpoint inhibitor (ICI) therapy in low-risk patients.

In our study, we identified two biomarkers by estimating amino acid and immune status in gliomas on the basis of the expression of mRNA. PSMD3, also known as P58 or RPN3, is one of the member in proteasome subunit S3 family, which acts as the non-ATPase subunits of the $19 S$ regulator lid[34]. PSMD3 is widely expressed in most tissues and defined as oncogene in various cancers. WBC and neutrophil counts are related with the expression of PSMD3. Additionally, PSMD3 is also related with glucose-related features of carbohydrates and fatty acids from diet $[35,36]$. Besides, the higher level of PSMD3 mRNA predicts worse prognosis of acute myeloid leukemia patients and PSMD3 promotes the progression of chronic myeloid leukemia by stabilizing NF-kB $[37,38]$. Consistently, PSMD3 is upregulated in breast cancer compared with normal tissue and patients with higher expression level of PSMD3 is related with worse survival. And PSMD3 is strongly associated with the expression of HER2, which can stabilize HER2 from degradation [39]. PSMC5 is defined as a 19S regulatory component and it could identify and transform ubiquitin labeled proteins into form of degradation which can be mediated by 20S complex [40]. Interestingly, PSMC5 directly regulates transcription. For instance, it can influence the activity class II trans-activator to regulate the transcription of MHC class II [41], besides, it can also recruit p53 to the promoter of p21 to upregulate its expression, which can decrease the DNA damage mediated by ultraviolet [42]. Yet, the specific functions of both biomarkers in gliomas still remain unclear, more researches are needed in further study.

Finally, several limitations of our work should be mentioned including (1) although two biomarkers have been identified, the potential function of these genes still remains unclear and should be explored in the future study; (2) tumor heterogeneity is one of the most important feature in gliomas [43], which also means different microenvironment features exist among diverse tumor sites. Yet all of the data and information are collected from public database, which makes it impossible to detect the immune status in the same or diverse tumor regions. As a result, this gene signature should be better validated in the well- designed, multicenter, prospective studies

\section{Conclusions}

In a summary, the construction and validation of 12 amino acid metabolism and immune-related genes has been defined as a prognostic signature. This prognosis signature can predict the prognosis of patients and help to select the individualized therapeutic strategy in clinical practice, which provides a comprehensive perspective for clarifying the underlying mechanisms that determine the prognosis for glioma. In addition, our risk score model is associated with the immune status of glioma patients, which may imply the potential effect of immunotherapy. Besides, we also have identified PSMC5 and PSMD3 as new biomarkers in glioma.

\section{Abbreviations}

PD-L1: programmed cell death 1 ligand; IDO: indolamine 2,3-dioxygenase; TGF- $\beta$ : transforming growth factor $\beta$; TCGA: the cancer genome atlas; CGGA: Chinese Glioma Genome Atlas; ssGSEA: single sample gene sets enrichment analysis; WGCNA: weighted gene co-expression network analysis; LASSO: least absolute shrinkage and selection operator; ICl: immune checkpoint inhibitor

\section{Declarations}

\section{Authors' contributions}

Qianxue Chen and Fanen Yuan designed the research. Yang Xu, Liguo Ye and Rongxin Geng carried out the work. Ping Hu, Qian Sun,and Shiao Tong analysed the data and wrote the paper. All authors read and approved the final manuscript.

\section{Author details}

1. Department of Neurosurgery, Renmin Hospital of Wuhan University, Wuhan 430060, China. 


\section{Acknowledgements}

We gratefully acknowledge The Cancer Genome Atlas pilot project and Chinese Glioma Genome Atlas, which made the genomic data and clinical data of glioma available.

\section{Competing interests}

The authors declare that they have no conflict of interest.

\section{Data availabity}

Publicly available datasets were analyzed in this study. This data can be found below:1.https://www.cancer.gov/ 2.http://www.cgga.org.cn/; The datasets used and/or analyzed during the current study are available from the corresponding author on reasonable request.

\section{Consent for publication}

Not applicable.

\section{Ethics approval and consent to participate}

The following information was supplied related to ethical approvals (i.e., approving body and any reference numbers):

Institutional Ethics Committee of the Faculty of Medicine at Renmin Hospital of Wuhan University approval (2012LKSZ (010) H) to carry out the study within its facilities

\section{Funding}

This work was supported by the National Natural Science Foundation of China (No.82072764).

\section{References}

1. Dang CV. Links between metabolism and cancer. Genes Dev. 2012;26:877-90.

2. S S, Mg VH. Emerging Roles for Branched-Chain Amino Acid Metabolism in Cancer [Internet]. Cancer cell. Cancer Cell; 2020 [cited 2021 Mar 2]. Available from: https://pubmed.ncbi.nlm.nih.gov/32049045/.

3. Lemos H, Huang L, Prendergast GC, Mellor AL. Immune control by amino acid catabolism during tumorigenesis and therapy. Nat Rev Cancer. 2019;19:162-75.

4. Guccione E, Richard S. The regulation, functions and clinical relevance of arginine methylation. Nat Rev Mol Cell Biol. 2019;20:642-57.

5. Greene LI, Bruno TC, Christenson JL, D’Alessandro A, Culp-Hill R, Torkko K, et al. A Role for Tryptophan-2,3-dioxygenase in CD8 T-cell Suppression and Evidence of Tryptophan Catabolism in Breast Cancer Patient Plasma. Mol Cancer Res. 2019;17:131-9.

6. Opitz CA, Litzenburger UM, Sahm F, Ott M, Tritschler I, Trump S, et al. An endogenous tumour-promoting ligand of the human aryl hydrocarbon receptor. Nature. 2011;478:197-203.

7. Fallarino F, Grohmann U, Hwang KW, Orabona C, Vacca C, Bianchi R, et al. Modulation of tryptophan catabolism by regulatory T cells. Nat Immunol. 2003;4:1206-12.

8. Commisso C, Davidson SM, Soydaner-Azeloglu RG, Parker SJ, Kamphorst JJ, Hackett S, et al. Macropinocytosis of protein is an amino acid supply route in Ras-transformed cells. Nature. 2013;497:633-7.

9. Ostrom QT, Patil N, Cioffi G, Waite K, Kruchko C, Barnholtz-Sloan JS. CBTRUS Statistical Report: Primary Brain and Other Central Nervous System Tumors Diagnosed in the United States in 2013-2017. Neuro Oncol. 2020;22:iv1-96.

10. Tan AC, Ashley DM, López GY, Malinzak M, Friedman HS, Khasraw M. Management of glioblastoma: State of the art and future directions. CA Cancer J Clin. 2020;70:299-312.

11. Lim M, Xia Y, Bettegowda C, Weller M. Current state of immunotherapy for glioblastoma. Nat Rev Clin Oncol. 2018;15:422-42. 
12. Wainwright DA, Balyasnikova IV, Chang AL, Ahmed AU, Moon K-S, Auffinger B, et al. IDO expression in brain tumors increases the recruitment of regulatory T cells and negatively impacts survival. Clin Cancer Res. 2012;18:6110-21.

13. Bloch O, Crane CA, Kaur R, Safaee M, Rutkowski MJ, Parsa AT. Gliomas promote immunosuppression through induction of B7-H1 expression in tumor-associated macrophages. Clin Cancer Res. 2013;19:3165-75.

14. Colombo MP, Piconese S. Regulatory-T-cell inhibition versus depletion: the right choice in cancer immunotherapy. Nat Rev Cancer. 2007;7:880-7.

15. Liberzon A, Birger C, Thorvaldsdóttir H, Ghandi M, Mesirov JP, Tamayo P. The Molecular Signatures Database (MSigDB) hallmark gene set collection. Cell Syst. 2015;1:417-25.

16. Miranda A, Hamilton PT, Zhang AW, Pattnaik S, Becht E, Mezheyeuski A, et al. Cancer stemness, intratumoral heterogeneity, and immune response across cancers. Proc Natl Acad Sci U S A. 2019;116:9020-9.

17. Yoshihara K, Shahmoradgoli M, Martínez E, Vegesna R, Kim H, Torres-Garcia W, et al. Inferring tumour purity and stromal and immune cell admixture from expression data. Nat Commun. 2013;4:2612.

18. Tibshirani R. The lasso method for variable selection in the Cox model. Stat Med. 1997;16:385-95.

19. Heagerty PJ, Lumley T, Pepe MS. Time-dependent ROC curves for censored survival data and a diagnostic marker. Biometrics. 2000;56:337-44.

20. Newman AM, Liu CL, Green MR, Gentles AJ, Feng W, Xu Y, et al. Robust enumeration of cell subsets from tissue expression profiles. Nat Methods. 2015;12:453-7.

21. Gene Ontology Consortium. The Gene Ontology (G0) project in 2006. Nucleic Acids Res. 2006;34:D322-326.

22. Shannon P, Markiel A, Ozier O, Baliga NS, Wang JT, Ramage D, et al. Cytoscape: a software environment for integrated models of biomolecular interaction networks. Genome Res. 2003;13:2498-504.

23. Wu Z, Liu Y, Xu J, Xie J, Zhang S, Huang L, et al. A Ventilator-associated Pneumonia Prediction Model in Patients With Acute Respiratory Distress Syndrome. Clin Infect Dis. 2020;71:S400-8.

24. Lapointe S, Perry A, Butowski NA. Primary brain tumours in adults. Lancet. 2018;392:432-46.

25. Jackson CM, Choi J, Lim M. Mechanisms of immunotherapy resistance: lessons from glioblastoma. Nat Immunol. 2019;20:1100-9.

26. Liu Y-Q, Chai R-C, Wang Y-Z, Wang Z, Liu X, Wu F, et al. Amino acid metabolism-related gene expression-based risk signature can better predict overall survival for glioma. Cancer Sci. 2019;110:321-33.

27. Kesarwani P, Prabhu A, Kant S, Chinnaiyan P. Metabolic remodeling contributes towards an immune-suppressive phenotype in glioblastoma. Cancer Immunol Immunother. 2019;68:1107-20.

28. Li Z, Zhang H. Reprogramming of glucose, fatty acid and amino acid metabolism for cancer progression. Cell Mol Life Sci. 2016;73:37792.

29. Timosenko E, Hadjinicolaou AV, Cerundolo V. Modulation of cancer-specific immune responses by amino acid degrading enzymes. Immunotherapy. 2017;9:83-97.

30. Li B, Chan HL, Chen P Immune Checkpoint Inhibitors: Basics and Challenges. Curr Med Chem. 2019;26:3009-25.

31. Cloughesy TF, Mochizuki AY, Orpilla JR, Hugo W, Lee AH, Davidson TB, et al. Neoadjuvant anti-PD-1 immunotherapy promotes a survival benefit with intratumoral and systemic immune responses in recurrent glioblastoma. Nat Med. 2019;25:477-86.

32. Adams S, Teo C, McDonald KL, Zinger A, Bustamante S, Lim CK, et al. Involvement of the kynurenine pathway in human glioma pathophysiology. PLoS One. 2014;9:e112945.

33. Liu Y, Liang X, Dong W, Fang Y, Lv J, Zhang T, et al. Tumor-Repopulating Cells Induce PD-1 Expression in CD8 + T Cells by Transferring Kynurenine and AhR Activation. Cancer Cell. 2018;33:480-494.e7.

34. Okada Y, Kamatani Y, Takahashi A, Matsuda K, Hosono N, Ohmiya H, et al. Common variations in PSMD3-CSF3 and PLCB4 are associated with neutrophil count. Hum Mol Genet. 2010;19:2079-85.

35. Arnett ZJ-S, Parnell DK, Lee LD, Ma Y-C, Smith Y CE, et al. Genetic variants at PSMD3 interact with dietary fat and carbohydrate to modulate insulin resistance. J Nutr 2013;143:354-61.

36. lio E, Matsuura K, Nishida N, Maekawa S, Enomoto N, Nakagawa M, et al. Genome-wide association study identifies a PSMD3 variant associated with neutropenia in interferon-based therapy for chronic hepatitis C. Hum Genet. 2015;134:279-89.

37. Dai Y-J, Hu F, He S-Y, Wang Y-Y. Epigenetic landscape analysis of IncRNAs in acute myeloid leukemia with DNMT3A mutations. Ann Transl Med. 2020;8:318.

38. Bencomo-Alvarez AE, Rubio AJ, Olivas IM, Gonzalez MA, Ellwood R, Fiol CR, et al. Proteasome 26S subunit, non-ATPases 1 (PSMD1) and 3 (PSMD3), play an oncogenic role in chronic myeloid leukemia by stabilizing nuclear factor-kappa B. Oncogene 2021;40:2697-710. 
39. Chen FAS, Ho L-C, Cheng Y-S, Liu T-C, Chang Y-R H-L, et al. Proteasome 26S Subunit, non-ATPase 3 (PSMD3) Regulates Breast Cancer by Stabilizing HER2 from Degradation. Cancers (Basel). 2019;11:E527.

40. Yun YJ-H, Lee HS, Baek S-J, Lee J-H, Song C-W J-Y, et al. Radiosensitizing effect of PSMC5, a 19S proteasome ATPase, in H460 lung cancer cells. Biochem Biophys Res Commun 2016;469:94-100.

41. Bhat KP, Turner JD, Myers SE, Cape AD, Ting JP-Y, Greer SF. The 19S proteasome ATPase Sug1 plays a critical role in regulating MHC class II transcription. Mol Immunol. 2008;45:2214-24.

42. Qe QZGWJYSP, Ma W E-M, et al. The ubiquitin-proteasome system regulates p53-mediated transcription at p21waf1 promoter. Oncogene [Internet]. Oncogene; 2007 [cited 2021 Jul 8];26. Available from: https://pubmed.ncbi.nlm.nih.gov/17224908/.

43. Puchalski RB, Shah N, Miller J, Dalley R, Nomura SR, Yoon J-G, et al. An anatomic transcriptional atlas of human glioblastoma. Science. 2018;360:660-3.

\section{Figures}
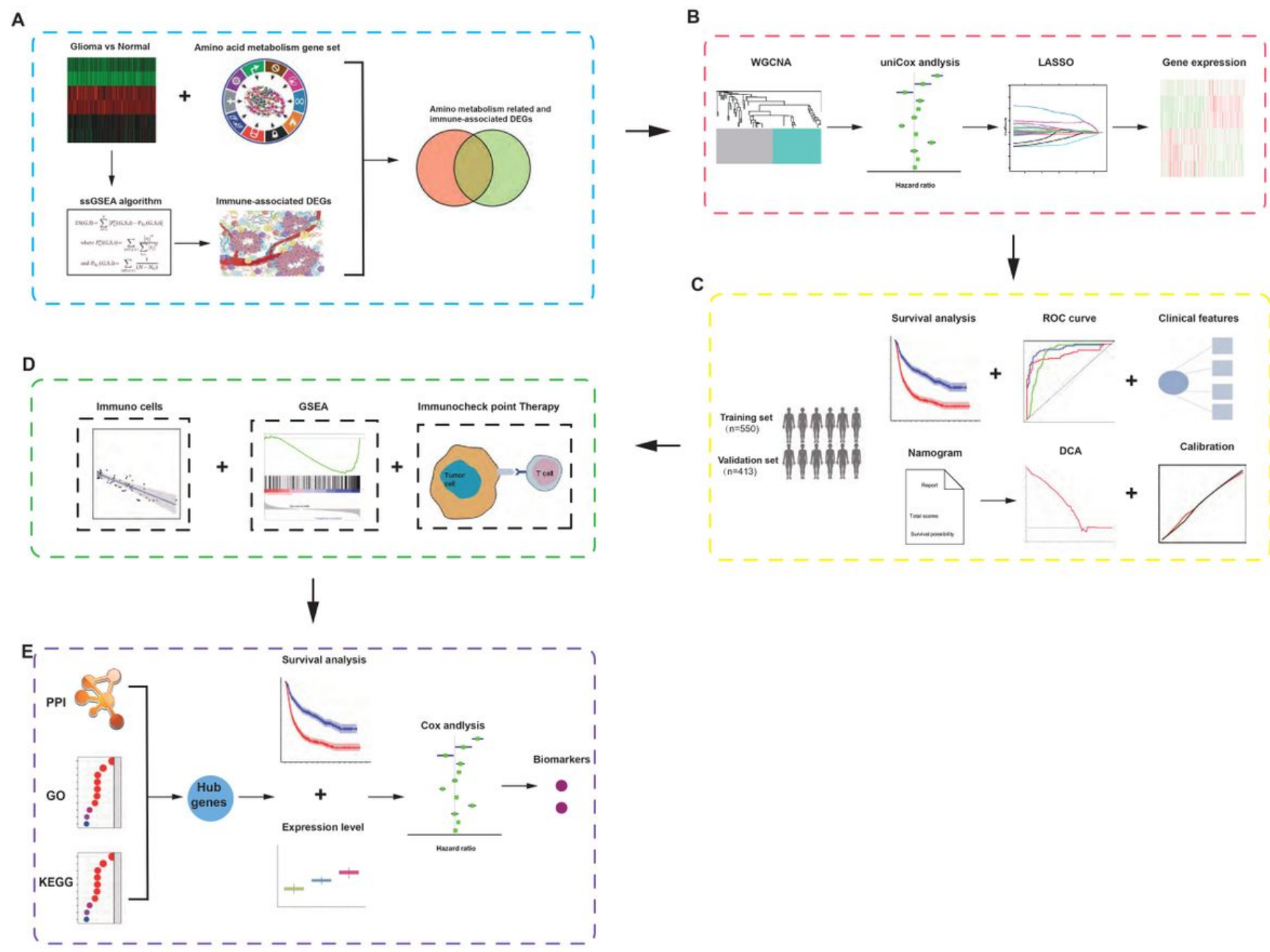

\section{Figure 1}

Schematic diagram of the study design. (A) Identification of amino-acid metabolism related and immune associated gene module in glioma patients among various hallmarks of cancer. (B) WGCNA and LASSO Cox algorithms were combined to develop an amino-acid metabolism related and immune associated gene signature for prognosis. (C) The prognostic and predictive capacities were validated in different cohorts and methods. (D) Comprehensive analyses of enriched pathways, immune cell infiltration and therapeutic responses in different risk groups.

(E) Identification of hub genes and biomarkers from gene signature for glioma. 
A

Gene dendrogram and module colors
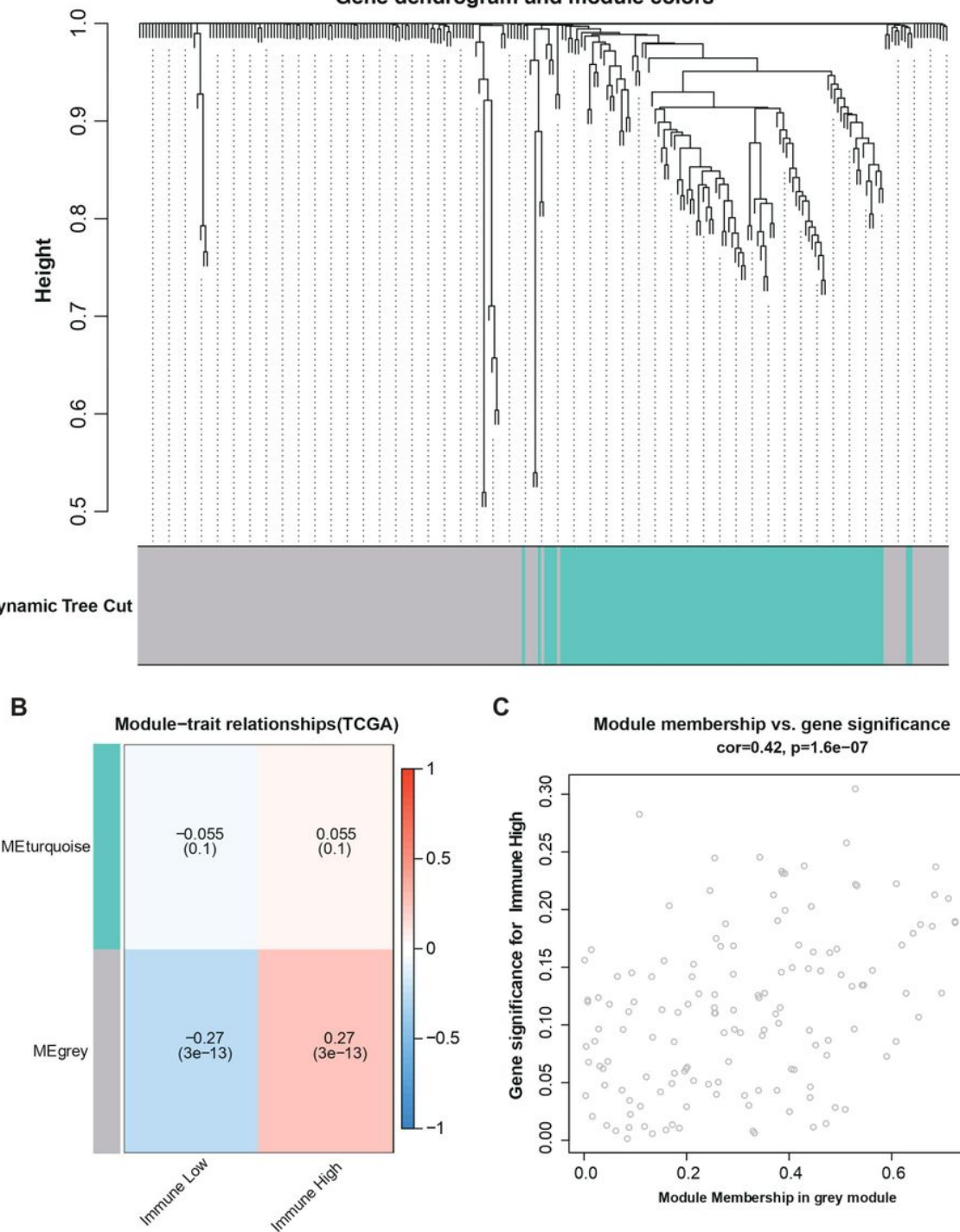

C
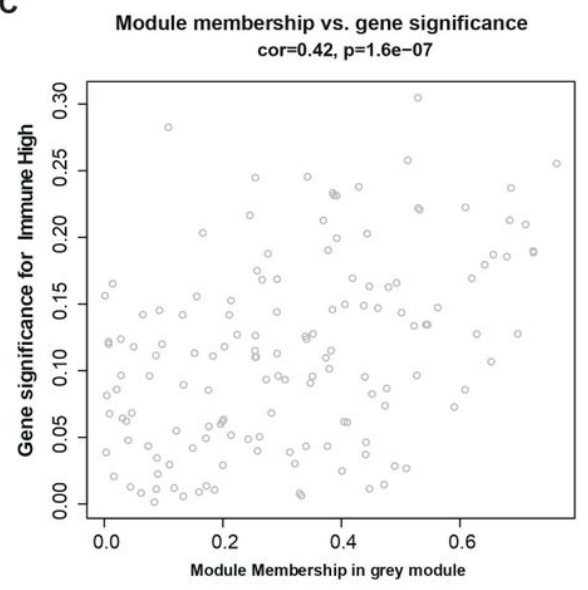

Figure 2

Identification of modules associated with the immunity in the TCGA-glioma dataset. (A) The cluster dendrogram of co-expression network modules were ordered by a hierarchical clustering of genes based on the 1-TOM matrix. Each module was assigned different colors. (B) Module-immune relationships. Each row corresponds to a color module and column corresponds to immune score (high and low). (C) Module membership vs. gene significance in grey module. 
A

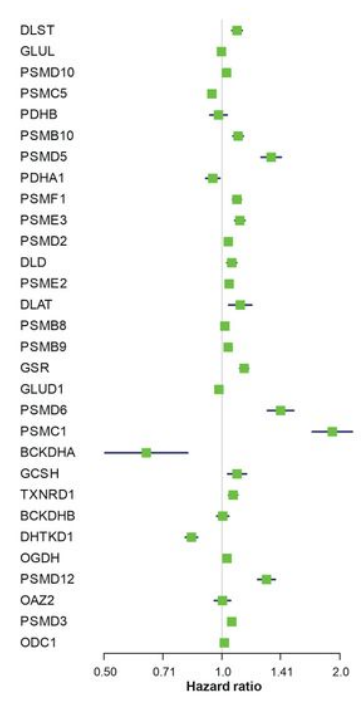

B

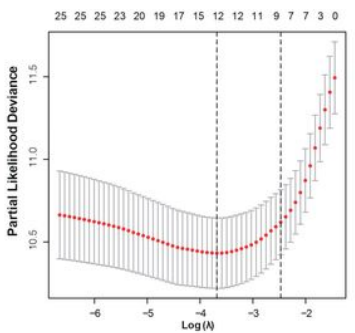

C

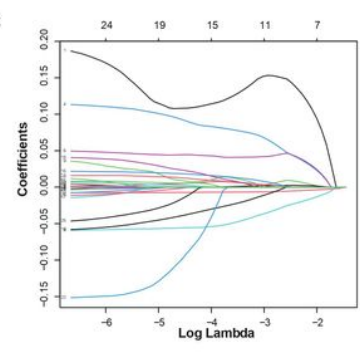

D

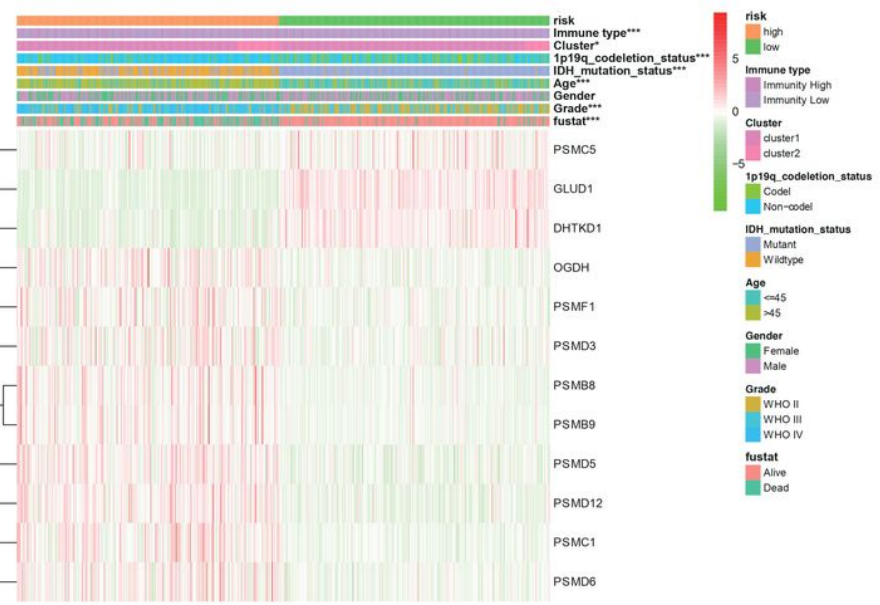

Figure 3

Identification of 12-gene risk signature for OS by LASSO regression analysis in CGGA datasets. (A) 30 genes associated with OS of patients with gliomas by univariate Cox regression analysis. (B) Red dots represent average partial likelihood deviances for every model with a given lambda, and vertical bars indicate the upper and lower values of the partial likelihood deviance errors. The vertical black dotted lines define the optimal values of lambda, which provides the best fit. Survival curves of patients in high-risk group and low risk group of The Cancer Genome Atlas (TCGA) glioma cohort. (C) The selection of the tuning parameter (lambda) in the LASSO model by tenfold cross-validation based on minimum criteria for OS; the lower $X$ axis shows log (lambda), and the upper $X$ axis shows the average number of OS-genes. The $Y$ axis indicates partial likelihood deviance error. (D) Heat map shows the association of risk scores and clinic-pathological features based on the 12-gene risk signature. 

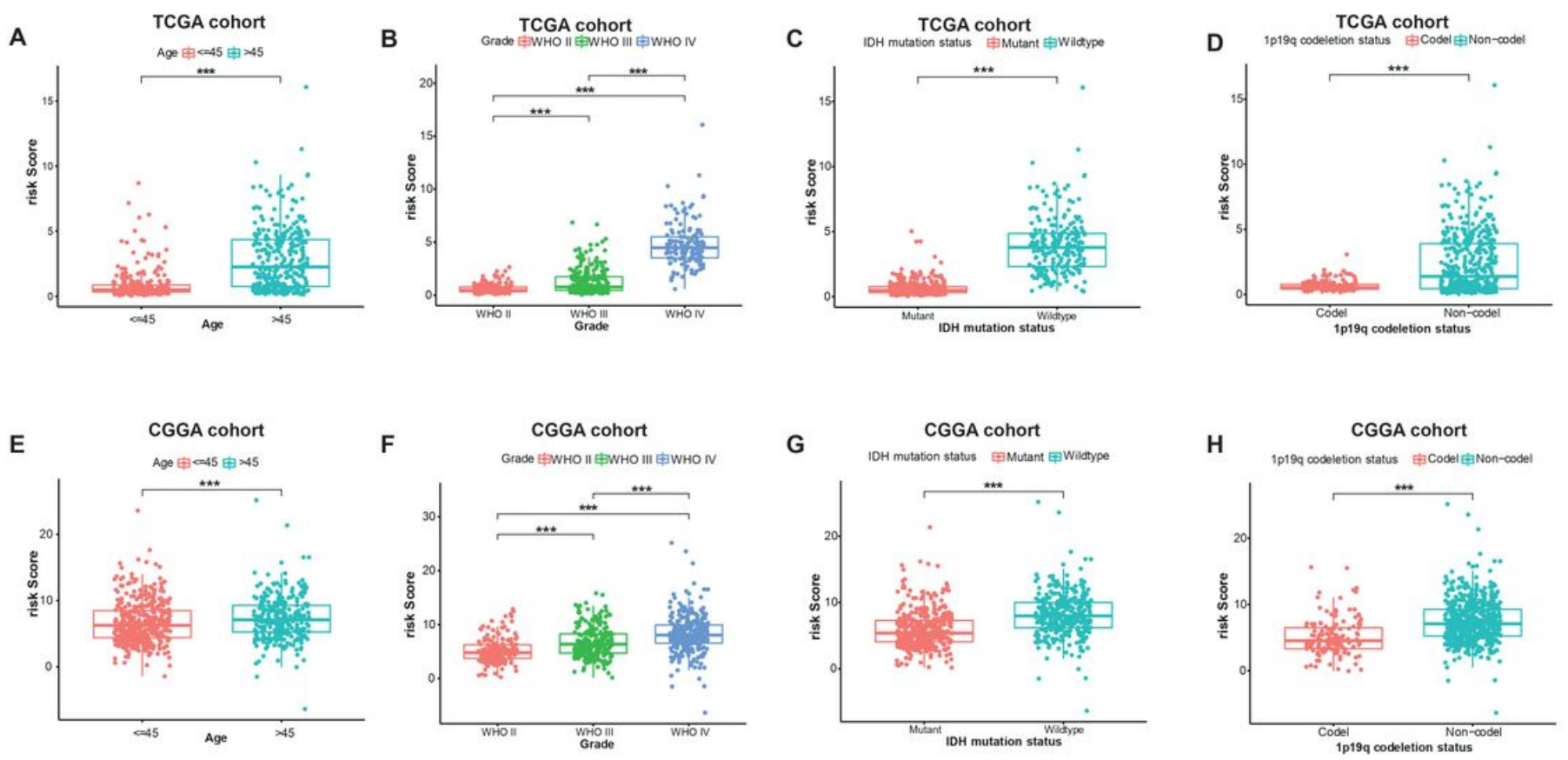

\section{Figure 4}

Associations between the amino acid-related and immune associated signature and other features in both TCGA and CGGA datasets. Distribution of the amino acid-related and immune associated gene signature in patients stratified by age (A, E), WHO grade (B, F), IDH1 status in each grade $(C, G), 1 p / 19 q$ status $(D, H),{ }^{\star \star \star} P<0.001$. IDH, isocitrate dehydrogenase; TCGA, The Cancer Genome Atlas; CGGA, Chinese Glioma Genome Atlas; Codel, codeletion; GBM, glioblastoma; WHO, World Health Organization 
A

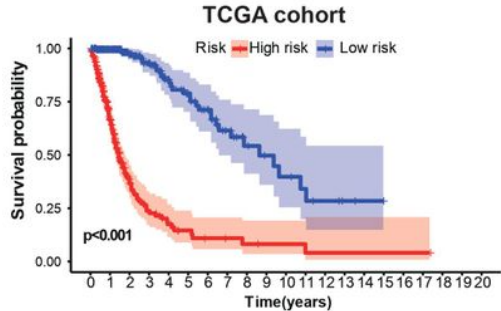

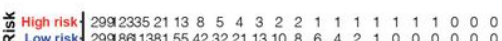
$\frac{1}{01234567891011121314151617181920}$ Time(years)

C

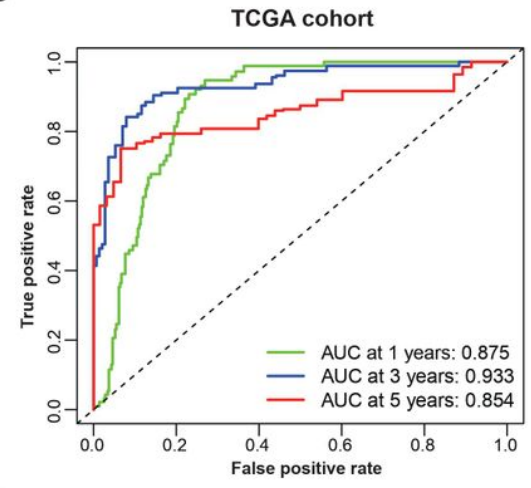

E
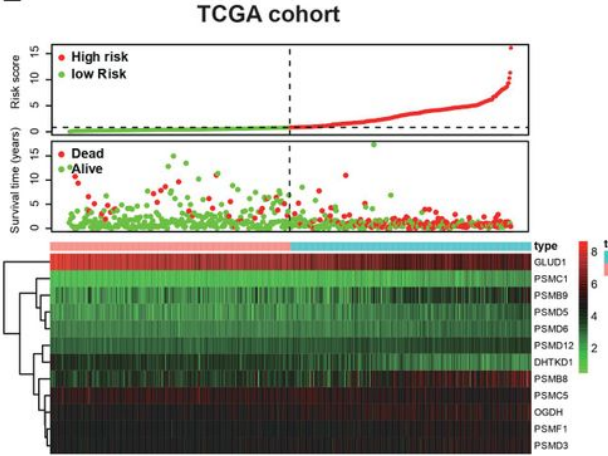

CGGA cohort

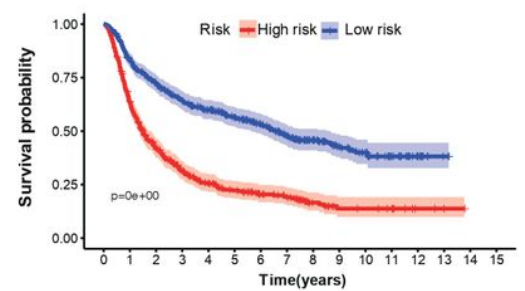

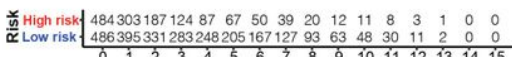
$\frac{1}{6} \begin{aligned} & 13456 \\ & 6\end{aligned}$

D
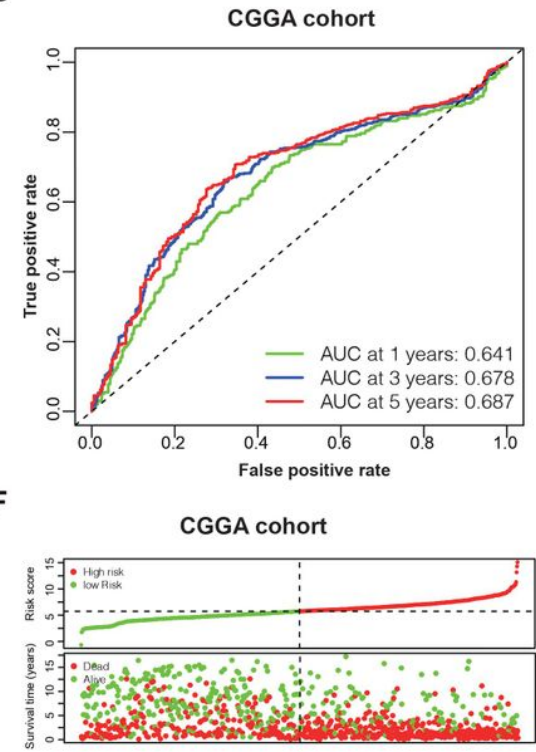

(a)

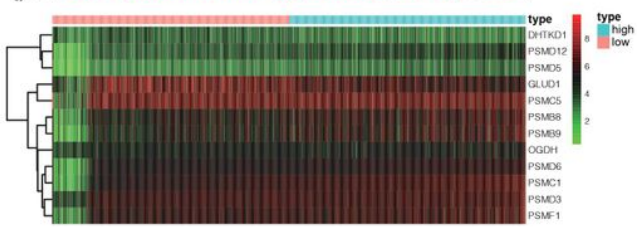

Figure 5

Development of the Risk Score Signature and Assessment of the Predicting Capacity (A-B) Survival curves of patients in high-risk group and low risk group of TCGA glioma and CGGA. Patients in high-risk group suffered shorter overall survival; (C-D) show survival-dependent receiver operating characteristic (ROC) curves validation at 1, 3,5-year of prognostic value of the prognostic index in the two databases (TCGA and CGGA, respectively); (E-F) Distribution of risk score, overall survival (OS), gene expression in TCGA and CGGA databases were also shown. Distribution of risk score, OS and heat map of the expression of twelve genes in low-risk and high-risk groups are listed in the picture from top to bottom. 
A

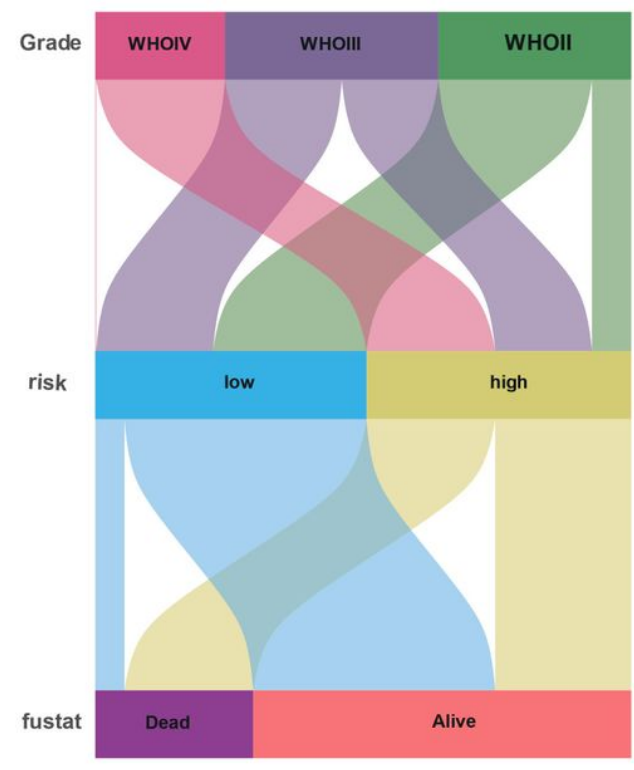

B

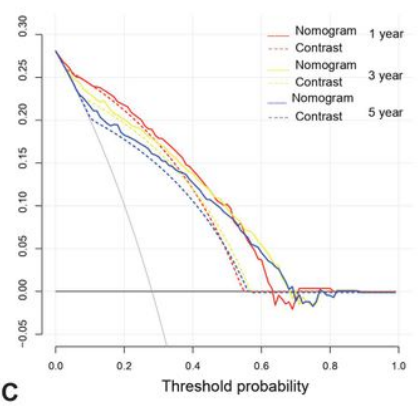

C

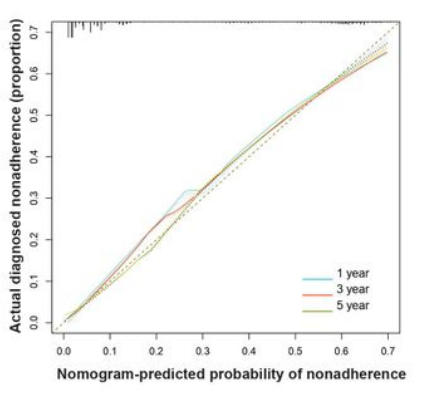

D

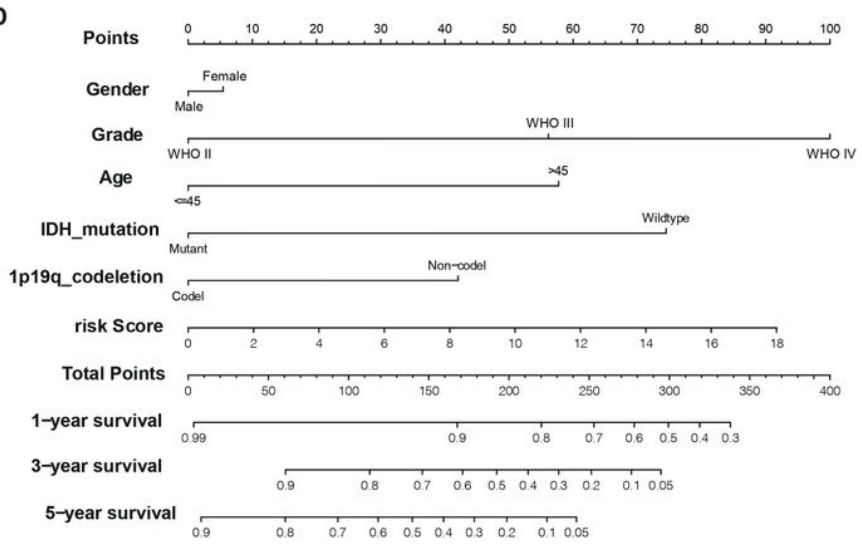

Figure 6

Combination of the risk signature and clinicopathological features improves risk stratification and survival prediction (A). A decision tree was constructed to improve risk stratification. (B) Decision curve analysis of the nomogram and contrast system for predicting 1-, 3-, and 5-year survival rate of patients. (C) Calibration analysis indicated a high accuracy of survival prediction. (D) A nomogram was constructed to quantify risk assessment for individual patients. 
A

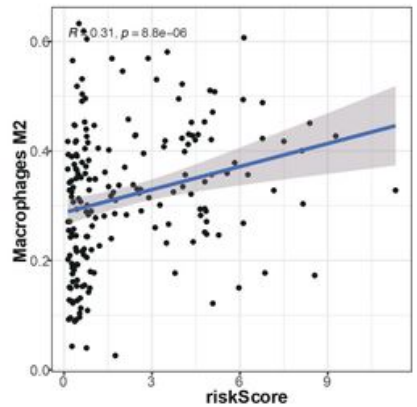

E

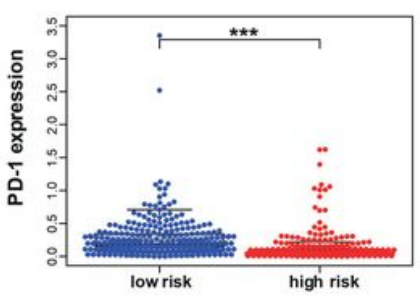

B

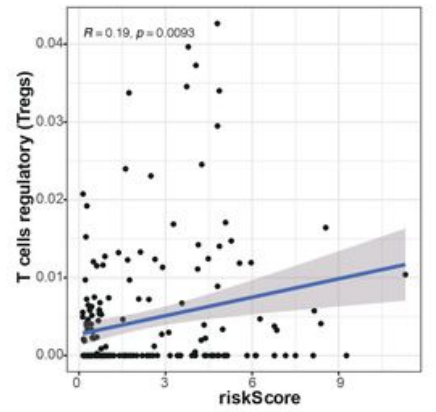

F

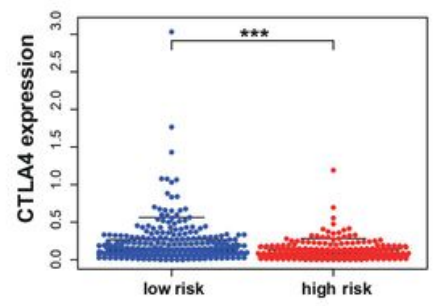

C

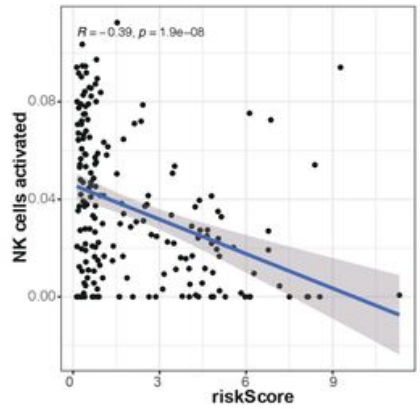

G

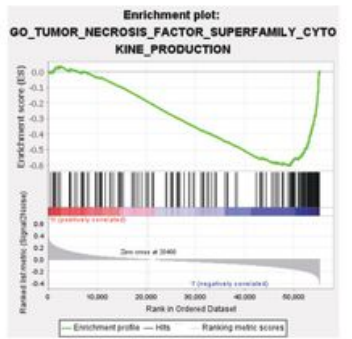

D

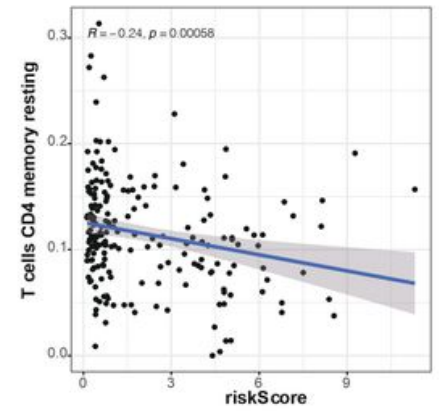

H

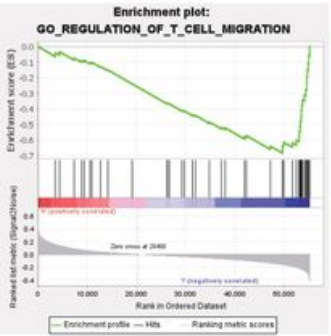

Figure 7

The Differences in Immunocyte Infiltration Degree and enrichment plots of immune related gene sets from gene set enrichment analysis between High- and Low-Risk TCGA Cohorts (A-D) The correlation with immunocyte infiltration was performed by using Pearson correlation analysis. M2; Tregs; NK cells; CD4+T cells. (E, F) The correlation with immune-checkpoint expression. PD-1; CTLA4. (G, H) GSEA analysis revealing immune-related biological processes correlated with the signature. 
A

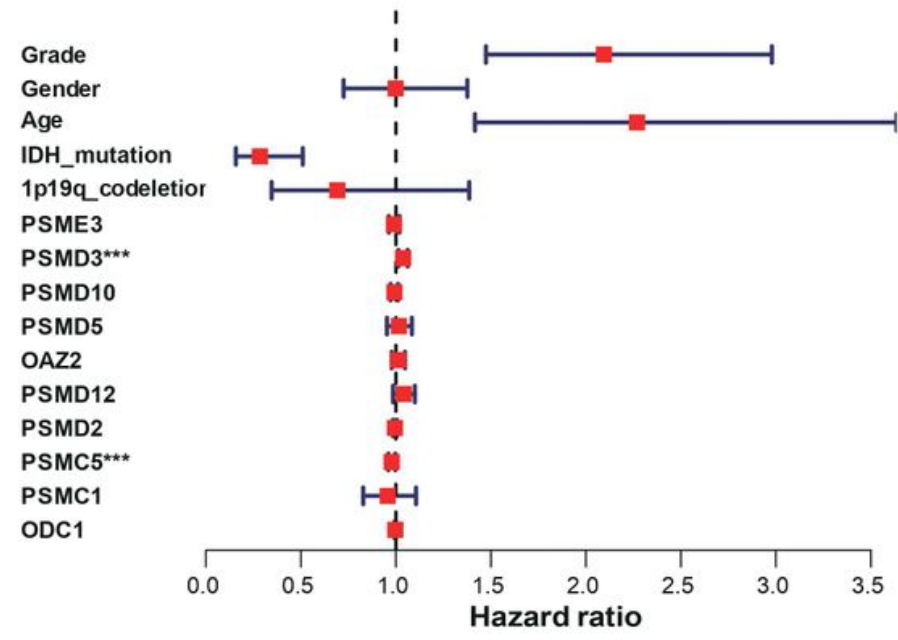

B

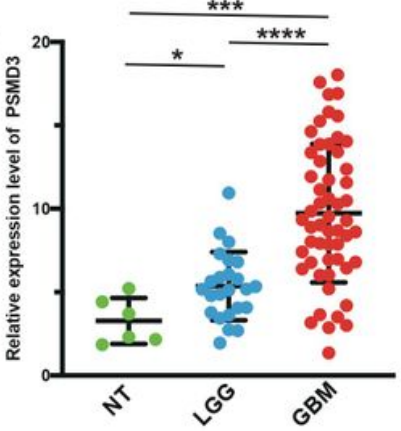

C

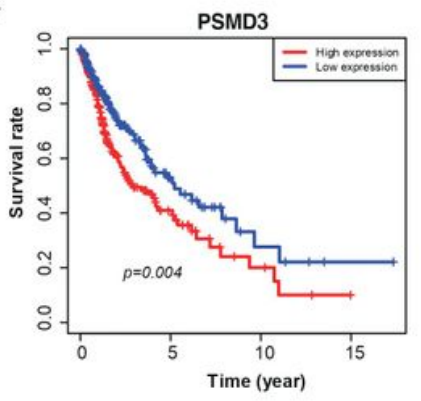

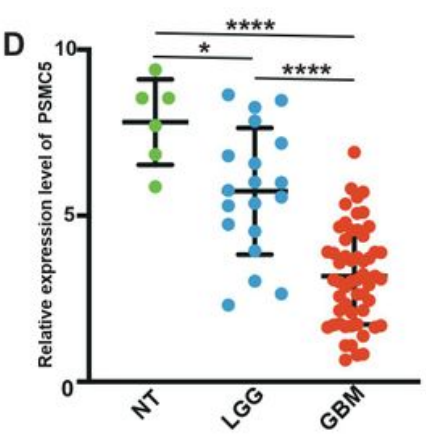

$\mathbf{E}$

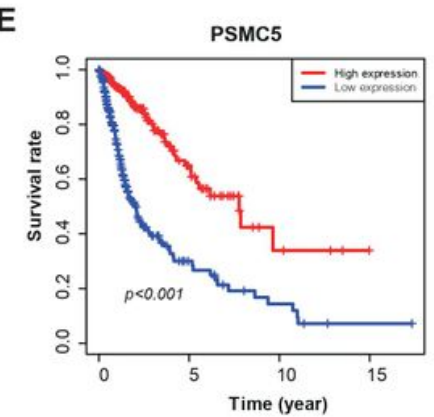

\section{Figure 8}

Identification of hub genes of amino metabolism related-risk signature. (A) Forest plot of the multivariable Cox regression analysis of the effect of ten hub genes and clinicopathological variables on the OS of glioma patients. (B) The relative expression level of PSMD3 in normal, LGG and GBM tissues according to the rt-PCR results. (C) The OS survival analysis of PSMD3 in TCGA-glioma. (D) The relative expression level of PSMC5 in normal, LGG and GBM tissues according to the rt-PCR results. (E) The OS survival analysis of PSMC5 in TCGA-glioma.

\section{Supplementary Files}

This is a list of supplementary files associated with this preprint. Click to download.

- FigureS1.pdf

- Figures2.pdf

- Figures3.pdf

- Figures4.pdf

- FigureS5.pdf

- Tables1.docx

- Tables2.docx

- Tables3.docx 